\title{
Cerberus 2001 Team Description
}

\author{
H. Levent Akın ${ }^{1}$, Andon Topalov ${ }^{2}$, and Okyay Kaynak ${ }^{3}$
}

${ }^{1}$ Department of Computer Engineering, Boğaziçi University, 80815 İstanbul Turkey akin@boun.edu.tr

2 Department of Control Systems at the Technical University of Sofia Plovdiv Branch, Plovdiv, Bulgaria

3 Department of Electrical and Electronics Engineering, Boğaziçi University, 80815 İstanbul Turkey kaynak@boun.edu.tr

\begin{abstract}
Cerberus is one of the four new teams in the RoboCup Tournament, Legged Robot Category. The team is a joint effort of Boğaziçi University (Turkey) and Technical University of Sofia, Branch Plovdiv (Bulgaria). We have divided the robot software design into six major tasks, and worked on those tasks in parallel. The tasks were locomotion, localization, vision, behavior control, learning and communication. With limited time in our hands, our aims were a clear object oriented design, readable behavior architecture, and maximum code reuse.
\end{abstract}

\section{Introduction}

Cerberus is the first international team participating in Sony's Legged Robot League within RoboCup 2001. This has implied some difficulties in its organization: there where some difficulties to explain thoroughly the ideas each group had in mind because of the necessity to use a foreign language (English) and use predominantly e-mails to maintain communication between the team members. Making an international team has also some advantages: the possibility to use the know-how and the previous research developments in both countries.

The challenge of designing a robotic soccer team is best met with a lot of hands-on experience. The noisy real-time environment, the dynamic nature of the opponents, the different lighting conditions all necessitate robust algorithms for the robot and require arduous fine-tuning.

Our general strategy was to divide the robot software into six major tasks, and assign one team for each task. The tasks were locomotion, localization, vision, behavior control, learning and communication. Locomotion and vision were low-level tasks that involved interfacing the sensors and actuators of the AIBO robot via middleware. Localization relies on the information from vision and locomotion to determine robot's position. Learning was used whenever necessary. The behavior control involves the design of a conceptual finite state machine to guide the behavior of the robot. Cooperation and communication can be seen as integrated parts of the behavior. 


\section{Development Team}

The team is a joint effort of Boğaziçi University (Turkey) and Technical University of Sofia, Branch Plovdiv (Bulgaria). The head of the Cerberus project is Prof. Okyay Kaynak, faculty member at the Electrical and Electronics Department of Boğaziçi University and is the UNESCO chair on Mechatronics.

\subsection{Turkish Team}

Assoc. Prof. H. Levent Akın, the head of the Artificial Intelligence Laboratory at the Department of Computer Engineering, leads the Turkish team. The Turkish team consists of the following students: Hatice Köse, Çiğdem Gündüz, Olcay Taner Yıldız, Ersin Başaran, Albert Ali Salah, Önder Tombuş, Onur Dikmen and Tamer Demir.

\subsection{Bulgarian Team}

The Bulgarian team is lead by Assoc. Prof. Andon Venelinov Topalov from the Department of Control Systems at the Technical University of Sofia Plovdiv Branch. The other members of the team are Christo Iliev Radev, Petya Emilova Pavlova, Nikola Georgiev Shakev, Ivan Kavalchev, Jordan Kirilov Tombakov and Anastasia Christova Radeva.

\section{The Architecture}

The Cerberus team has developed a behavior-based architecture for the robots. The components of the architecture are described briefly below.

\section{$3.1 \quad$ Vision}

The Turkish team worked on the vision system. Decision trees and Multi Layer Perceptrons were compared for object recognition. Decision trees were found to be faster and more robust for the successful classification of colors. After classification, we implemented the region finding codes.

\subsection{Localization}

Localization relies on the recognition of beacons and odometry. A global map is kept where the position of the robot and other locations of interest are recorded probabilistically. Each image frame processed by the vision module contributes in updating the relative probabilities. 


\subsection{Planner and Behaviors}

There are two different planners for the goalkeeper and the attackers. The behavior module currently consists of some basic behaviors that can be switched by planner module in order to achieve more complex behaviors of the robotic players on the field and different team strategies during the game. The planner module is designed by the Turkish team. We use graph representations and algorithms to store the behavior finite state machine as shown in Figure 1.

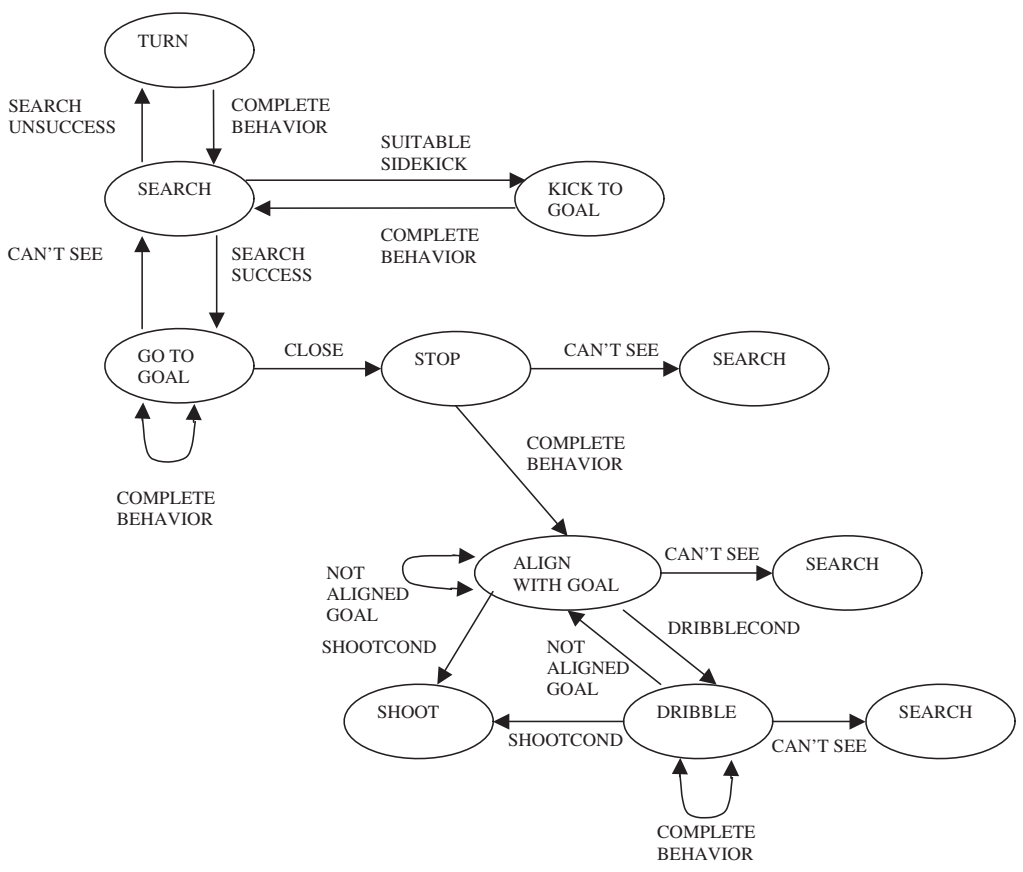

Fig. 1. Planner finite state diagram for the attacker

These behaviors are built by using the following low level behaviors: standing, lateral walking, turning around a specified rotation center, walking straight, dribbling, kicking, going to a specified point, goalkeeper standing, goalkeeper turning, goalkeeper walking forward and goalkeeper walking backward.

For reliable kicking and ball interception, the head control and locomotion are closely coupled with the vision module. For obstacle avoidance, we use an algorithm similar to the controller given in [1]. Some of the behaviors are directly implemented within the locomotion module (different kicking styles, walking straight, turning, etc.) and others like "going to a point" behavior, which is based on a genetically trained fuzzy-neural network [2], 3], are implemented in the behavior module. 
While active most of the behaviors receive feedback information about the changes in robot's coordinates and orientation approximately every 1.2 seconds. After completing each behavior a notification message is sent to the planner module. Planner module can decide to interrupt at any time the implementation of the currently running behavior.

\subsection{Locomotion}

The Bulgarian group of the team developed the locomotion module. The basic structure of the module and its interface is very close to the one developed by the Swedish team for RoboCup 2000 competitions [4. This structure has been adopted due to the limited time available and the command interface for passing the desired linear and angular velocity using robot's point model as data. This allows us to implement some behavior control approaches previously developed for wheeled mobile robots more easily.

\subsection{Communication and Multi-agent Behaviors}

The Bulgarian group of the team develops the communication modules. At this stage, the only way for the Sony's robotic dogs to communicate between them is by using their onboard speakers and stereo microphones.

The main goal of communications between the robotic dogs is to provide information exchange within the team to be used as a basis for multi-agent cooperation. There are currently two kinds of commands that are used in the communication: commands for cooperative play and localization. The commands contain the identification number of the sender and data.

\section{Acknowledgements}

Our work is supported by Boğaziçi University Foundation and Boğaziçi University Research Fund project 01A101.

\section{References}

1. Köse, H., Akın, H. L.: Towards a Robust Cognitive Architecture for Small Autonomous Mobile Robots ISCIS XV, The Fifteenth International Symposium on Computer and Information Sciences, October, 11-13, 2000, Istanbul, Turkey, pp.447-455, 2000 .

2. Topalov, A.V., Tzafestas, S.G.: Layered Multi-agent Reactive-behavior learning in a Robotic Soccer Proc. of the 6th IFAC Symposium on Robot Control, SYROCO'00, Vienna, September, 2000, pp.325-330.,2000.

3. Topalov, A.V., Tzafestas, S.G.: Fuzzy-neural-genetic Layered Multi-agent Reactive Control of a Robotic Soccer Accepted article for publication in the book: Data Mining for Design and Manufacturing: Methods and Applications, D. Braha editor, Kluwer Academic Publishers,. 2001.

4. Safiotti, A., et al.: Team Sweden in P.Stone, T.Balch, G.Kraetzchmar (Eds.), Robocup 2000: Robot Soccer, World Cup IV, pp.643-646, Springer-Verlag, 2001. 